\title{
Noncommutative quantum field theory and gravity
}

\author{
Konstantinos Anagnostopoulos • Paolo Aschieri • \\ Martin Bojowald • Harald Grosse - Larisa Jonke • \\ George Zoupanos
}

This special issue is dedicated to the proceedings of the "2nd School and Workshop on Quantum Gravity and Quantum Geometry” Corfu, September 13-20, 2009. The aim of the meeting, sponsored by the European Science Foundation network "Quantum Gravity and Quantum Geometry", was to gather experts in different approaches to

\footnotetext{
K. Anagnostopoulos · G. Zoupanos

Department of Physics, National Technical University of Athens,

School of Applied Mathematics and Physical Sciences,

Zografou Campus, 15780 Athens, Greece

e-mail: konstant@mail.ntua.gr

G. Zoupanos

e-mail: Zoupanos@mail.cern.ch

P. Aschieri $(\varangle)$

Dipartimento di Scienze e Tecnologie Avanzate, Facoltà di Scienze M.F.N., and I.N.F.N. Sezione di Torino, Gruppo collegato di Alessandria,

Viale T. Michel 11, 15121 Alessandria, Italy

e-mail: aschieri@mfn.unipmn.it; aschieri@to.infn.it
}

\section{Bojowald}

The Pennsylvania State University, 104 Davey Lab, 256,

University Park, PA 16802-6300, USA

e-mail: bojowald@gravity.psu.edu

H. Grosse

Faculty of Physics, University of Vienna,

Boltzmanngasse 5, 1090 Vienna, Austria

e-mail: harald.grosse@univie.ac.at

L. Jonke

Theoretical Physics Division, Rudjer Boskovic Institute,

P.O. Box 180, 10002 Zagreb, Croatia

e-mail: larisa@irb.hr 
the study of the geometry of quantum spacetime, i.e., the geometry arising from (or needed for) quantum gravity and underlying also quantum field theories as well as strings. A main motivation being that the knowledge of these mathematical structures leads to advances in the different formulations of a consistent quantum gravity theory. In this perspective introductory courses to loop quantum gravity and to renormalization were presented, and spacetime has been studied as a spin foam model, as a dynamical triangulation, as a noncommutative space, and as a matrix space. A common underlying feature in these approaches is that of a discretized space-time structure, implemented through triangulations or noncommutativity of the algebra of functions. Despite the appearance of these central themes in several different approaches, the precise implementations can often differ considerably from one another. Collecting a large set of representative discussions in this special issue should thus be beneficial for a wide community.

Among the topics presented are the kinematical properties, including quantum group symmetries, of noncommutative spacetime structures relevant in gravity, in string theory and membrane theory on one hand, and dynamical issues such as the formulation of field theories on noncommutative spacetime and their quantum properties, like ultraviolet-infrared duality and renormalization on the other hand. Loop quantum gravity, in the context of its kinematical representation, has led to several results about noncommutativity and other non-trivial spacetime structures. Especially in recent years new constructions have been performed which were discussed at the workshop. These include models in $2+1$ dimensions with noncommutative connections, and Fourier transforms on spaces of connections. Spin-foam models and group field theory provide a link to higher form fields and aspects of renormalization.

In higher gauge theories the gauge potential is a 2-form (a 1-form in loop space) or a higher form. This topic is also relevant in order to understand the geometry and the dynamics of extended objects (like branes). Higher gauge theory also arises as categorification of the usual gauge theory of particles. This special issue starts indeed with lecture notes on higher form fields and then continues with noncommutative/quantum gravity and field theories contributions.

There were five school lecturers: Abhay Ashtekar, John Baez, John Barrett, Vincent Rivasseau, Carlo Rovelli.

The 22 seminar speakers were: Paolo Aschieri, Glenn Barnich, Aristid Baratin, Chong-Sun Chu, Denjoe O'Connor, Brian Dolan, Winston Fairbairn, Steven Giddings, Kristina Giesel, Harald Grosse, Jens Hoppe, Jerzy Lukierski, Fedele Lizzi, Jerzy Lewandowski, Shahn Majid, Catherine Meusburger, Martin Reuter, Peter Schupp, Harold Steinacker, Richard Szabo, Jochum van der Bij, Satoshi Watamura.

The Scientific Organizers were: John Barrett, Harald Grosse, Larisa Jonke, George Zoupanos.

We sincerely thank everybody who contributed to the success of this school and workshop that took place as one of the activities within the Corfu Summer Institute "Schools and Workshops on Elementary Particle Physics and Gravity" (CORFU2009), website: http://www.physics.ntua.gr/corfu2009/. In particular we thank the many (about 100) young students that came long ways from different countries. Special thanks are due to all speakers and the organizers, the conference secretary Mrs. Ifigenia Moraiti, the school officer Mrs. Rachel Avramidou and the group of our 
graduate students who helped in various ways and contributed in a very significant manner to the success of the meeting. Finally, we wish to express our gratitude to the "European Science Foundation" and especially to the "Quantum Geometry and Quantum Gravity ESF Network” our main sponsor whose financial contribution made it all possible. 